\title{
LOCAL EXACT CONTROLLABILITY OF THE DIFFUSION EQUATION IN ONE DIMENSION
}

\author{
MARIUS BECEANU
}

Received 21 December 2002

This paper establishes the local exact null controllability of the diffusion equation in one dimension using distributed controls in the case of the Dirichlet boundary value problem. Most of the techniques used in the course of the proof are borrowed from Barbu (2002).

\section{Formulation of the problem}

Consider the diffusion equation

$$
\begin{gathered}
y_{t}-(a(y))_{x x}=m u, \quad \forall(x, t) \in Q=I \times(0, T), \\
y(x, t)=0, \quad \forall(x, t) \in \Sigma=\partial I \times(0, T), \\
y(x, 0)=y_{0}, \quad x \in I,
\end{gathered}
$$

where $I=\left(i_{1}, i_{2}\right)$ is a bounded real interval and $m$ is the characteristic function of an open subset $\omega$ of $I$.

The ellipticity of this operator is insured by the condition $0<\mu \leq a^{\prime}(x)$, for all $x \in \mathbb{R}$. We need $\left|a^{\prime}(x)\right|,\left|a^{\prime \prime}(x)\right|$, and $\left|a^{\prime \prime \prime}(x)\right| \leq M<\infty$ as well in order to establish the desired controllability result.

Equation (1.1) is said to be exactly null controllable if there is $u \in L^{2}(Q)$ such that $y(T) \equiv 0$, where $y \in C\left([0, T] ; L^{2}(I)\right)$ is the solution to (1.1).

We will first establish some controllability results for the linearized equation

$$
\begin{gathered}
y_{t}-\left(a^{\prime}(\tilde{y}) y_{x}\right)_{x}=m u, \quad \forall(x, t) \in Q, \\
y(x, t)=0, \quad \forall(x, t) \in \Sigma, \\
y(x, 0)=y_{0}, \quad x \in I,
\end{gathered}
$$

where $\tilde{y}$ is some function with $\tilde{y}_{x}, \sqrt{t} \tilde{y}_{t} \in L^{\infty}(Q), \tilde{y}_{x t} \in L^{2}(Q)$, and $\left.\tilde{y}\right|_{\Sigma}=0$. Henceforth, $a^{\prime}(\tilde{y})$ will be denoted by $b$. 
In order to establish the controllability of (1.2), it is sufficient to prove a Carleman-type inequality concerning the dual system

$$
\begin{gathered}
p_{t}+\left(b p_{x}\right)_{x}=g, \quad \forall(x, t) \in Q, \\
p(x, t)=0, \quad \forall(x, t) \in \Sigma, \\
p(x, T)=p_{T}, \quad x \in I .
\end{gathered}
$$

However, this only solves the problem for the linearized equation. Our purpose will be to eventually show that we can make $y=\tilde{y}$ in (1.2), since then the controllability of this equation would also imply that of the diffusion equation (1.1).

We will arrive at the desired conclusion by means of the Kakutani fixed-point theorem and Pontryagin's principle. Indeed, under strong regularity conditions for the initial data $\left(\left(a\left(y_{0}\right)\right)_{x x},\left(y_{0}\right)_{x}\right.$, and $y_{0}$ belonging to $L^{2}(I)$ and of sufficiently small norm), we will see that the multifunction $y=\Phi(\tilde{y})$, where $y$ is any solution to (1.2), takes an $L^{2}(Q)$-compact set into itself. The other conditions for applying Kakutani's theorem are easily met.

The final step will be to prove that we can dispense with at least some of the conditions on the initial data, due to the regularizing properties of the diffusion equation. We are going to show that, for every $y_{0} \in H^{1}(I)$ of sufficiently small norm, the diffusion equation is null controllable.

\section{The Carleman inequality}

In the following, we will assume the function $a$ to be fixed, and therefore we will not mention the constants $\mu$ and $M$ explicitly. However, we will keep track of $\tilde{y}$ and its derivatives.

In the course of the proof, we are going to need the following lemma.

Lemma 2.1. There exists a function $\psi \in C^{2}(\bar{I})$ such that $\psi(x)>0$ for all $x \in I$, $\psi(x)=0$ on $\partial I$, and $\left|\psi_{x}(x)\right|>0$ for all $x \in \bar{I} \backslash \omega_{0}$, where $\omega_{0}$ is an open set such that $\overline{\omega_{0}} \subset I$.

The proof is obvious (see [1] for a more general case).

Throughout the paper, we will use a fixed $\psi$ and a fixed $\overline{\omega_{0}} \subset \omega$.

In addition, we define, for any $\lambda>0$, the functions $\alpha$ and $\phi: Q \rightarrow \mathbb{R}$ by

$$
\alpha(x, t)=\frac{e^{\lambda \psi(x)}-e^{2 \lambda\|\psi\|_{C(\bar{I})}}}{t(T-t)}, \quad \phi(x, t)=\frac{e^{\lambda \psi(x)}}{t(T-t)} .
$$

Note that $\phi(x, t) \geq c>0$ for all $(x, t) \in Q$, and $e^{\delta \alpha} \phi^{k} \leq C<\infty$ for all $\delta>0, k \in \mathbb{R}$.

We are now ready to formulate the Carleman inequality concerning (1.3). The proof below is, by many ways, identical to the one given by Imanuvilov in a different case (see [2]). 
Theorem 2.2. For any solution $p$ of the dual system (1.3), with $\left\|b_{x}\right\|_{L^{\infty}(Q)}$, $\left\|\sqrt{t} b_{t}\right\|_{L^{\infty}(Q)} \leq \rho$ and for every $\lambda \geq \lambda_{0}(\rho), s \geq s_{0}(\lambda)$, the following inequality holds:

$$
\begin{aligned}
& \int_{Q} e^{2 s \alpha}\left(s^{3} \phi^{3} p^{2}+s \phi p_{x}^{2}+s^{-1} \phi^{-1}\left(p_{t}^{2}+p_{x x}^{2}\right)\right) \\
& \leq C(\lambda, \rho)\left(\int_{Q_{\omega}} e^{2 s \alpha} s^{3} \phi^{3} p^{2}+\int_{Q} e^{2 s \alpha} g^{2}\right),
\end{aligned}
$$

where $C(\lambda, \rho)$ is a constant independent of $p, g$, and $s$, but which may depend on $\psi$, $\lambda$, and $\rho$, and $Q_{\omega}=\omega \times(0, T)$.

Proof. This proof follows step by step the one given for [1, Theorem 1.2.1] (the Carleman inequality concerning the heat equation [1, pages 145-152]).

By taking $z=e^{s \alpha} p$ in the dual system (1.3), we obtain

$$
\begin{aligned}
& z_{t}-s \alpha_{t} z+\left(b z_{x}\right)_{x}-2 s \lambda \phi \psi_{x} \cdot b z_{x}+\left(s^{2} \lambda^{2} \phi^{2} \psi_{x}^{2}-s \lambda^{2} \phi \psi_{x}^{2}-s \lambda \phi \psi_{x x}\right) b z \\
& \quad-s \lambda \phi \psi_{x} b_{x} z=e^{s \alpha} g \\
& z(0)=z(T)=0 \quad \text { on } I \\
& \left.z\right|_{\Sigma}=0 .
\end{aligned}
$$

If we set

$$
\begin{aligned}
& B(t) z=-\left(b z_{x}\right)_{x}-\left(s^{2} \lambda^{2} \phi^{2} \psi_{x}^{2}+s \lambda^{2} \phi \psi_{x}^{2}\right) b z+s \alpha_{t} z \\
& X(t) z=-2 s \lambda^{2} \phi \psi_{x}^{2} b z-2 s \lambda \phi \psi_{x} \cdot b z_{x} \\
& Z(t) z=s \lambda \phi \psi_{x} b_{x} z+s \lambda \phi \psi_{x x} b z
\end{aligned}
$$

the equation can be rewritten as $z_{t}-B(t) z+X(t) z=Z(t) z+e^{s \alpha} g$. Then, starting with the relation

$$
\begin{aligned}
\frac{d}{d t} \int_{I} B(t) z \cdot z d x & =\int_{I} B(t) z_{t} \cdot z+B(t) z \cdot z_{t}+B_{t}(t) z \cdot z d x \\
& =2 \int_{I} B(t) z\left(B(t) z-X(t) z+Z(t) z+e^{s \alpha} g\right) d x+\int_{I} B_{t}(t) z \cdot z d x
\end{aligned}
$$

and integrating it on $(0, T)$, we obtain

$$
2 \int_{Q}(B(t) z)^{2}+2 Y=-2 \int_{Q} B(t) z\left(Z(t) z+e^{s \alpha} g\right)-\int_{Q} B_{t}(t) z \cdot z,
$$

where $Y=-\int_{Q} B \cdot X$, that is,

$$
Y=-\int_{Q}\left(2 s \lambda \phi \psi_{x} \cdot b z_{x}+2 s \lambda^{2} \phi \psi_{x}^{2} b z\right) \cdot\left(\left(b z_{x}\right)_{x}+\left(s^{2} \lambda^{2} \phi^{2} \psi_{x}^{2}+s \lambda^{2} \phi \psi_{x}^{2}\right) b z-s \alpha_{t} z\right) .
$$


796 Local exact controllability of the diffusion equation in 1D

Then, we evaluate

$$
\int_{Q} B_{t}(t) z \cdot z=\int_{Q} b_{t} z_{x}^{2}-\int_{Q}\left(s^{2} \lambda^{2} \phi^{2} \psi_{x}^{2} b+s \lambda^{2} \phi \psi_{x}^{2} b\right)_{t} z^{2}+s \alpha_{t t} z^{2} .
$$

Set $\gamma(\lambda)=e^{2 \lambda\|\psi\|_{C(T)}}$ and we take $s \geq \gamma(\lambda)$ and $\lambda \geq \lambda_{0}$. In addition, put

$$
D(s, \lambda, z)=\int_{Q} s^{3} \lambda^{3} \phi^{3} z^{2}+s \lambda \phi z_{x}^{2}
$$

We eventually obtain that

$$
\left|\int_{Q} B_{t}(t) z \cdot z\right| \leq C\left(1+\left\|\phi^{-1} b_{t}\right\|_{L^{\infty}(Q)}\right) D(s, \lambda, z) .
$$

Furthermore,

$$
\begin{aligned}
& 2\left|\int_{Q} B(t) z\left(Z(t) z+e^{s \alpha} g\right)\right| \\
& \quad \leq \int_{Q}(B(t) z)^{2}+C\left(1+\left\|\phi^{-1} b_{x}^{2}\right\|_{L^{\infty}(Q)}\right) D(s, \lambda, z)+C \int_{Q} e^{2 s \alpha} g^{2} .
\end{aligned}
$$

From (2.6), (2.10), and (2.11), we obtain

$$
Y \leq C\left(1+\left\|\sqrt{t} b_{t}\right\|_{L^{\infty}(Q)}+\left\|b_{x}\right\|_{L^{\infty}(Q)}^{2}\right) D(s, \lambda, z)+C \int_{Q} e^{2 s \alpha} g^{2} .
$$

On the other hand, we have the following inequalities that will give lower estimates:

$$
\left|\int_{Q}\left(2 s \lambda \phi \psi_{x} \cdot b z_{x}+s \lambda \phi \psi_{x} b_{x} z\right) s \alpha_{t} z\right| \leq C D(s, \lambda, z)
$$

for $s \geq \gamma(\lambda)$; then

$$
\begin{aligned}
& \left|\int_{Q} s \lambda \phi \psi_{x} b_{x} z \cdot s \alpha_{t} z\right| \leq C|| b_{x} \|_{L^{\infty}(Q)} \cdot D(s, \lambda, z), \\
& \left|\int_{Q}\left(s \lambda^{2} \phi \psi_{x}^{2} b z\right) s \alpha_{t} z\right| \leq C D(s, \lambda, z) .
\end{aligned}
$$

Moreover,

$$
\begin{aligned}
-\int_{Q} s \lambda^{2} \phi \psi_{x}^{2} b z \cdot\left(b z_{x}\right)_{x} & =\int_{Q} s \lambda^{2}\left(\phi \psi_{x}^{2} b z\right)_{x} \cdot b z_{x} \\
& \geq \int_{Q} s \lambda^{2} \phi \psi_{x}^{2} b^{2} z_{x}^{2}-C\left(1+\left\|b_{x}\right\|_{L^{\infty}(Q)}^{2}\right) D(s, \lambda, z) .
\end{aligned}
$$


Furthermore,

$$
\begin{aligned}
&-\int_{Q}\left(2 s \lambda \phi \psi_{x} \cdot b z_{x}\right)\left(s^{2} \lambda^{2} \phi^{2} \psi_{x}^{2}+s \lambda^{2} \phi \psi_{x}^{2}\right) b z \\
& \quad=\int_{Q}\left(s^{3} \lambda^{3} \phi^{3} \psi_{x}^{3} b^{2}+s^{2} \lambda^{3} \phi^{2} \psi_{x}^{3} b^{2}\right)_{x} \cdot z^{2} \\
& \quad \geq \int_{Q}\left(3 s^{3} \lambda^{4} \phi^{3} \psi_{x}^{4}+2 s^{2} \lambda^{4} \phi^{2} \psi_{x}^{4}\right) \cdot b^{2} z^{2}-C\left(1+\left\|b_{x}\right\|_{L^{\infty}(Q)}\right) D(s, \lambda, z) .
\end{aligned}
$$

Finally,

$$
-\int_{Q} 2 s \lambda \phi \psi_{x} \cdot b z_{x}\left(b z_{x}\right)_{x} \geq-\int_{\Sigma} s \lambda \phi \frac{d \psi}{d \nu} \cdot b^{2} z_{x}^{2} d \sigma-C D(s, \lambda, \phi) .
$$

Since $\psi>0$ on $I$ and $\psi=0$ on $\partial I$, we have $d \psi / d \nu \leq 0$, and therefore

$$
-\int_{\Sigma} s \lambda \phi \frac{d \psi}{d \nu} \cdot b^{2} z_{x}^{2} d \sigma \geq 0
$$

Combining inequalities (2.13), (2.14), (2.15), (2.16), and (2.17), we obtain

$$
Y \geq \int_{Q} s^{3} \lambda^{4} \phi^{3} \psi_{x}^{4} b^{2} z^{2}+s \lambda^{2} \phi \psi_{x}^{2} b^{2} z_{x}^{2}-C\left(1+\left\|b_{x}\right\|_{L^{\infty}(Q)}^{2}\right) D(s, \lambda, z) .
$$

From (2.19) and (2.12), we get

$$
\begin{aligned}
\int_{Q} s^{3} & \lambda^{4} \phi^{3} \psi_{x}^{4} b^{2} z^{2}+s \lambda^{2} \phi \psi_{x}^{2} b^{2} z_{x}^{2} \\
\leq & C\left(1+\left\|\sqrt{t} b_{t}\right\|_{L^{\infty}(Q)}+\left\|b_{x}\right\|_{L^{\infty}(Q)}^{2}\right) D(s, \lambda, z)+C \int_{Q} e^{2 s \alpha} g^{2} \\
& \leq C(\rho) D(s, \lambda, z)+C \int_{Q} e^{2 s \alpha} g^{2} .
\end{aligned}
$$

Because $b \geq \mu$ and $\left|\psi_{x}\right| \geq c$ on $I \backslash \omega_{0}$, by making $\lambda$ sufficiently large, it follows that

$$
\begin{aligned}
& \int_{Q} s^{3} \lambda^{4} \phi^{3} z^{2}+s \lambda^{2} \phi z_{x}^{2} \\
& \quad \leq C(\rho) \int_{Q_{\omega_{0}}} s^{3} \lambda^{4} \phi^{3} z^{2}+s \lambda^{2} \phi z_{x}^{2}+C \int_{Q} e^{2 s \alpha} g^{2} .
\end{aligned}
$$

Hence, we can obtain, by exactly the methods used in [1, page 151], that

$$
\begin{aligned}
& \int_{Q} e^{2 s \alpha} s^{3} \lambda^{4} \phi^{3} p^{2}+s \lambda^{2} \phi p_{x}^{2} \\
& \quad \leq C(\rho) \int_{Q_{\omega_{0}}} e^{2 s \alpha} s^{3} \lambda^{4} \phi^{3} p^{2}+s \lambda^{2} \phi p_{x}^{2}+C \int_{Q} e^{2 s \alpha} g^{2} .
\end{aligned}
$$


Choose $\chi \in C_{0}^{\infty}(\omega)$ such that $\chi=1$ in $\omega_{0}$. If we multiply (1.3) by $e^{2 s \alpha} \chi \phi p$ and integrate on $Q$, we obtain that

$$
\begin{aligned}
\int_{Q} e^{2 s \alpha} \chi \phi b p_{x}^{2} & =-\int_{Q}\left(e^{2 s \alpha} \chi \phi\right)_{x} \cdot b p p_{x}+\int_{Q} e^{2 s \alpha} \chi \phi p g+\int_{Q} \chi\left(e^{2 s \alpha} \phi\right)_{t} p^{2} \\
& \leq C\left(1+\left\|b_{x}\right\|_{L^{\infty}(Q)}\right) \int_{Q_{\omega}} e^{2 s \alpha} s^{2} \lambda^{2} \phi^{3} z^{2}+C \int_{Q} e^{2 s \alpha} g^{2}
\end{aligned}
$$

Consequently, we get

$$
\int_{Q} e^{2 s \alpha} s^{3} \lambda^{4} \phi^{3} p^{2}+s \lambda^{2} \phi p_{x}^{2} \leq C(\rho) \int_{Q_{\omega}} e^{2 s \alpha} s^{3} \lambda^{4} \phi^{3} p^{2}+C \int_{Q} e^{2 s \alpha} g^{2} .
$$

Equivalently, we may say that, for $\lambda \geq \lambda_{0}(\rho)$ and $s \geq s_{0}(\lambda)$,

$$
\int_{Q} e^{2 s \alpha} s^{3} \phi^{3} p^{2}+s \phi p_{x}^{2} \leq C(\rho, \lambda) \int_{Q_{\omega}} e^{2 s \alpha} s^{3} \phi^{3} p^{2}+C \int_{Q} e^{2 s \alpha} g^{2} .
$$

Next, by squaring (1.3), multiplying it with $e^{2 s \alpha} s^{-1} \phi^{-1}$, and integrating on $Q$, we obtain

$$
\int_{Q} e^{2 s \alpha} s^{-1} \phi^{-1}\left(p_{t}+\left(b p_{x}\right)_{x}\right)^{2} \leq C \int_{Q} e^{2 s \alpha} g^{2}
$$

At the same time,

$$
\begin{aligned}
\int_{Q} e^{2 s \alpha} s^{-1} \phi^{-1} p_{t} \cdot\left(b p_{x}\right)_{x} \\
\quad=-\int_{Q}\left(e^{2 s \alpha} \phi^{-1} p_{t}\right)_{x} \cdot b p_{x} \\
\quad \geq \frac{1}{2} \int_{Q}\left(e^{2 s \alpha} \phi^{-1}\right)_{t} \cdot p_{x}^{2}-\frac{1}{2} \int_{Q} e^{2 s \alpha} s^{-1} \phi^{-1} p_{t}^{2}-C \int_{Q} s \lambda^{2} \phi p_{x}^{2} \\
\geq-\frac{1}{2} \int_{Q} e^{2 s \alpha} s^{-1} \phi^{-1} p_{t}^{2}-C \int_{Q} s \lambda^{2} \phi p_{x}^{2} .
\end{aligned}
$$

By (2.26) and (2.27), we obtain

$$
\int_{Q} e^{2 s \alpha} s^{-1} \phi^{-1}\left(p_{t}^{2}+\left(b p_{x}\right)_{x}^{2}\right) \leq C \int_{Q} e^{2 s \alpha} g^{2}+C \int_{Q} s \lambda^{2} \phi p_{x}^{2}
$$

Combining inequalities (2.25) and (2.28), we obtain the final result (2.2).

Proceeding as in [1, Corollary 1.2.1, page 145], one may obtain the following corollary. 
Corollary 2.3. Under the assumptions of Theorem 2.2, the following inequality holds:

$$
\int_{I} p^{2}(0) d x \leq C(s, \lambda, \rho)\left(\int_{Q_{\omega}} e^{2 s \alpha} s^{3} \phi^{3} p^{2}+\int_{Q} e^{2 s \alpha} g^{2}\right),
$$

where $C(s, \lambda, \rho)$ is a constant that does not depend on $p$ or $g$.

\section{Estimates for $y$}

First, we denote $Q_{t}=I \times(0, t)$ and $f=m u$. Thus, system (1.2) can be rewritten as

$$
\begin{aligned}
y_{t}-\left(b y_{x}\right)_{x}=f, & \forall(x, t) \in Q, \\
y(x, t)=0, & \forall(x, t) \in \Sigma, \\
y(x, 0)=y_{0}, & x \in I .
\end{aligned}
$$

We will prove a few estimates concerning the solution $y$ of system (3.1).

Multiplying (3.1) by $y_{t}$ and integrating on $Q_{t}$, we get

$$
\int_{Q_{t}} y_{t}^{2}+\int_{Q_{t}} \frac{d}{d t} b y_{x}^{2}(t) \leq C\left(\int_{Q_{t}} f^{2}+\int_{Q_{t}} b_{t} y_{x}^{2}\right),
$$

so

$$
\begin{aligned}
\int_{Q} y_{t}^{2} & +\sup _{t \in[0, T]} \int_{I} y_{x}^{2}(t) d x \\
& \leq C\left(\int_{I} y_{x}^{2}(0) d x+\int_{Q} f^{2}+\int_{0}^{T} \frac{d t}{\sqrt{t}} \cdot\left\|\sqrt{t} b_{t}\right\|_{L^{\infty}(Q)} \cdot \sup _{t \in[0, T]} \int_{I} y_{x}^{2}(t) d x\right) \\
& \leq C_{0}\left(\int_{I} y_{x}^{2}(0) d x+\int_{Q} f^{2}+\left\|\sqrt{t} b_{t}\right\|_{L^{\infty}(Q)} \cdot \sup _{t \in[0, T]} \int_{I} y_{x}^{2}(t) d x\right) .
\end{aligned}
$$

Making $\left\|\sqrt{t} b_{t}\right\|_{L^{\infty}(Q)}$ sufficiently small $\left(\left\|\sqrt{t} b_{t}\right\|_{L^{\infty}(Q)} \leq 1 / 2 C_{0}\right)$, from (3.3) we infer that $y \in L^{\infty}\left((0, T) ; H_{0}^{1}(I)\right) \subset L^{\infty}(Q)$ and

$$
\|y\|_{L^{\infty}(Q)}^{2} \leq C \sup _{t \in[0, T]} \int_{I} y_{x}^{2}(t) d x \leq C\left(\int_{I} y_{x}^{2}(0) d x+\int_{Q} f^{2}\right) .
$$


As a consequence of the fact that $\left(b y_{x}\right)_{x}=y_{t}-f$, we also obtain from (3.3), when $\left\|t b_{t}\right\|_{L^{\infty}(Q)}$ is sufficiently small, that

$$
\int_{Q}\left(b y_{x}\right)_{x}^{2} \leq C\left(\int_{I} y_{x}^{2}(0) d x+\int_{Q} f^{2}\right)
$$

Multiplying (3.1) by $\left(b y_{x}\right)_{x t}$ and integrating on $Q_{t}$, we get

$$
\int_{Q_{t}} y_{t}\left(b y_{x}\right)_{x t}-\int_{Q_{t}}\left(b y_{x}\right)_{x}\left(b y_{x}\right)_{x t}=\int_{Q_{t}} f\left(b y_{x}\right)_{x t},
$$

and, by Green's formula,

$$
\int_{Q_{t}}\left(b y_{x t}^{2}+b_{t} y_{x} y_{x t}\right)+\frac{1}{2} \int_{Q_{t}} \frac{d}{d t}\left(b y_{x}\right)_{x}^{2}=-\int_{Q_{t}} f\left(b y_{x}\right)_{x t} .
$$

Through integration by parts, we obtain

$$
\begin{aligned}
& -\int_{Q_{t}} f\left(b y_{x}\right)_{x t} \\
& \quad=-\left.\int_{I} f\left(b y_{x}\right)_{x} d x\right|_{0} ^{t}+\int_{Q_{t}} f_{t}\left(b y_{x}\right)_{x} \\
& \quad \leq \int_{I}\left(f^{2}(0)+f^{2}(t)\right) d x+\frac{1}{4} \int_{I}\left(\left(b y_{x}\right)_{x}^{2}(0)+\left(b y_{x}\right)_{x}^{2}(t)\right) d x+\int_{Q_{t}}\left(b y_{x}\right)_{x}^{2}+\int_{Q_{t}} f_{t}^{2} \\
& \quad \leq \frac{1}{4} \int_{I}\left(\left(b y_{x}\right)_{x}^{2}(0)+\left(b y_{x}\right)_{x}^{2}(t)\right) d x+\int_{Q_{t}}\left(b y_{x}\right)_{x}^{2}+C \int_{Q_{t}}\left(f_{t}^{2}+f^{2}\right) .
\end{aligned}
$$

An elementary computation also proves that

$$
\begin{aligned}
\int_{Q_{t}} y_{x t} b_{t} y_{x} & \leq \frac{1}{4 c} \int_{Q_{t}} y_{x t}^{2}+c \int_{0}^{t}\left\|b_{t}(s)\right\|_{L^{\infty}(I)}^{2} d s \cdot \sup _{s \in[0, t]} \int_{I} y_{x}^{2}(s) d x \\
& \leq \frac{1}{4 c} \int_{Q_{t}} y_{x t}^{2}+C\left\|b_{x t}\right\|_{L^{2}(Q)}^{2} \cdot \sup _{s \in[0, T]} \int_{I} y_{x}^{2}(s) d x .
\end{aligned}
$$

Applying the preceding estimates into the equality (3.7) gives us

$$
\begin{aligned}
& \int_{Q_{t}} b y_{x t}^{2}+\int_{I}\left(b y_{x}\right)_{x}^{2}(t) d x \\
& \leq C\left(\int_{I}\left(b y_{x}\right)_{x}^{2}(0) d x+\int_{Q_{t}}\left(b y_{x}\right)_{x}^{2}+\int_{Q_{t}}\left(f_{t}^{2}+f^{2}\right)+\left\|b_{x t}\right\|_{L^{2}(Q)}^{2} \cdot \sup _{s \in[0, t]} \int_{I} y_{x}^{2}(s) d x\right) .
\end{aligned}
$$


Taking into account (3.4) and (3.5), for any $t \in[0, T]$, it is true that

$$
\begin{aligned}
\int_{Q} y_{x t}^{2} & +\int_{I}\left(b y_{x}\right)_{x}^{2}(t) d x \\
& \leq C\left(\int_{I}\left(b y_{x}\right)_{x}^{2}(0) d x+\int_{Q} f_{t}^{2}+\left(1+\left\|b_{x t}\right\|_{L^{2}(Q)}^{2}\right)\left(\int_{I} y_{x}^{2}(0) d x+\int_{Q} f^{2}\right)\right) .
\end{aligned}
$$

One consequence of this relation and of (3.4) is that $b y_{x} \in L^{\infty}\left((0, T) ; H^{1}(I)\right) \subset$ $L^{\infty}(Q)$, and we get the estimate

$$
\begin{aligned}
& \left\|y_{x}\right\|_{L^{\infty}(Q)}^{2} \\
& \quad \leq C\left(\int_{I}\left(b y_{x}\right)_{x}^{2}(0) d x+\int_{Q} f_{t}^{2}+\left(1+\left\|b_{x t}\right\|_{L^{2}(Q)}^{2}\right)\left(\int_{I} y_{x}^{2}(0) d x+\int_{Q} f^{2}\right)\right) .
\end{aligned}
$$

Taking the derivative of (3.1) with respect to $t$, we obtain

$$
y_{t t}-\left(b y_{t x}\right)_{x}-\left(b_{t} y_{x}\right)_{x}=f_{t}
$$

Multiplying this with $t y_{t t}$ and integrating on $Q_{t}$, we have

$$
\int_{Q_{t}} t y_{t t}^{2}+\int_{Q_{t}} t b y_{x t} y_{x t t}=\int_{Q_{t}} t\left(\left(b_{t} y_{x}\right)_{x}+f_{t}\right) y_{t t} .
$$

It follows that

$$
\int_{Q_{t}} t y_{t t}^{2}+\frac{1}{2} \int_{Q_{t}} \frac{d}{d t}\left(t b y_{x t}^{2}\right) \leq C\left(\int_{Q_{t}} t f_{t}^{2}+t\left(b_{t} y_{x}\right)_{x}^{2}+\int_{Q_{t}}\left(t b_{t}+b\right) y_{x t}^{2}\right) .
$$

Then, since $\left(b_{t} y_{x}\right)_{x}=b_{t x} y_{x}+b_{t} y_{x x}$,

$$
\begin{aligned}
\int_{Q_{t}} t\left(b_{t} y_{x}\right)_{x}^{2} & \leq 2\left(\int_{Q} t b_{t x}^{2} y_{x}^{2}+\int_{Q} t b_{t}^{2} y_{x x}^{2}\right) \\
& \leq C\left\|b_{x t}\right\|_{L^{2}(Q)}^{2} \cdot\left\|y_{x}\right\|_{L^{\infty}(Q)}^{2}+\left\|\sqrt{t} b_{t}\right\|_{L^{\infty}(Q)}^{2} \cdot \int_{Q} y_{x x}^{2}
\end{aligned}
$$

Furthermore, it is clearly true that

$$
\begin{aligned}
\int_{Q_{t}} t b_{t} y_{x t}^{2} & \leq \int_{0}^{t} \frac{d s}{\sqrt{s}} \cdot\left\|\sqrt{t} b_{t}\right\|_{L^{\infty}(Q)} \cdot \sup _{s \in[0, t]} \int_{I} s y_{x t}^{2}(s) d x \\
& \leq C\left\|\sqrt{t} b_{t}\right\|_{L^{\infty}(Q)} \cdot \sup _{t \in[0, T]} \int_{I} t y_{x t}^{2}(t) d x .
\end{aligned}
$$


It follows that

$$
\begin{aligned}
& \int_{Q} y_{t t}^{2}+\sup _{t \in[0, T]} \int_{I} t y_{x t}^{2}(t) d x \\
& \leq C_{1}\left(\int_{Q} f_{t}^{2}+\left\|y_{x}\right\|_{L^{\infty}(Q)}^{2} \cdot\left\|b_{x t}\right\|_{L^{2}(Q)}^{2}\right. \\
& \left.\quad+\left\|\sqrt{t} b_{t}\right\|_{L^{\infty}(Q)}^{2} \int_{Q} y_{x x}^{2}+\int_{Q} y_{x t}^{2}+\left\|\sqrt{t} b_{t}\right\|_{L^{\infty}(Q)} \cdot \sup _{t \in[0, T]} \int_{I} t y_{x t}^{2}(t) d x\right) .
\end{aligned}
$$

Since $b y_{x x}=\left(b y_{x}\right)_{x}-b_{x} y_{x}$, from (3.4) and (3.5) we can infer that

$$
\int_{Q} y_{x x}^{2} \leq C\left(1+\left\|b_{x}\right\|_{L^{\infty}(Q)}^{2}\right)\left(\int_{I} y_{x}^{2}(0) d x+\int_{Q} f^{2}\right) .
$$

Making this substitution, as well as those possible in virtue of (3.12) and (3.11), into (3.18), we get (for every $b$ with $\left\|\sqrt{t} b_{t}\right\|_{L^{\infty}(Q)} \leq 1 / 2 C_{1}$ )

$$
\begin{aligned}
& \int_{Q} y_{t t}^{2}+\sup _{t \in[0, T]} \int_{I} t y_{x t}^{2}(t) d x \\
& \leq C\left(\int_{Q} f_{t}^{2}+\left(\left\|b_{x t}\right\|_{L^{2}(Q)}^{2}+\left\|\sqrt{t} b_{t}\right\|_{L^{\infty}(Q)}^{2}\left(1+\left\|b_{x}\right\|_{L^{\infty}(Q)}^{2}\right)\right)\left(\int_{I} y_{x}^{2}(0) d x+\int_{Q} f^{2}\right)\right. \\
& \left.\quad+\int_{I}\left(b y_{x}\right)_{x}^{2}(0) d x+\int_{Q} f_{t}^{2}+\left(1+\left\|b_{x t}\right\|_{L^{2}(Q)}^{2}\right)\left(\int_{I} y_{x}^{2}(0) d x+\int_{Q} f^{2}\right)\right),
\end{aligned}
$$

which becomes (after sorting out the terms)

$$
\begin{aligned}
& \int_{Q} y_{t t}^{2}+\sup _{t \in[0, T]} \int_{I} t y_{x t}^{2}(t) d x \\
& \leq C\left(\int_{Q} f_{t}^{2}+\int_{I}\left(b y_{x}\right)_{x}^{2}(0) d x\right. \\
& \left.\quad+\left(1+\left\|b_{x t}\right\|_{L^{2}(Q)}^{2}+\left\|\sqrt{t} b_{t}\right\|_{L^{\infty}(Q)}^{2}\left(1+\left\|b_{x}\right\|_{L^{\infty}(Q)}^{2}\right)\right)\left(\int_{I} y_{x}^{2}(0) d x+\int_{Q} f^{2}\right)\right) .
\end{aligned}
$$

As a consequence, we see that $\sqrt{t} y_{t} \in L^{\infty}\left((0, T) ; H_{0}^{1}(I)\right) \subset L^{\infty}(Q)$ (with $\left\|\sqrt{t} y_{t}\right\|_{L^{\infty}(Q)}$ $\left.\leq C\left\|\sqrt{t} y_{x t}\right\|_{L^{\infty}\left((0, T) ; L^{2}(I)\right)}\right)$. 
Coming back to $\tilde{y}$ (recall that $b=a^{\prime}(\tilde{y})$ ), we note that

$$
\begin{aligned}
\left\|b_{x}\right\|_{L^{\infty}(Q)} & \leq C\left\|\tilde{y}_{x}\right\|_{L^{\infty}(Q)}, \quad\left\|\sqrt{t} b_{t}\right\|_{L^{\infty}(Q)} \leq C\left\|\sqrt{t} \tilde{y}_{t}\right\|_{L^{\infty}(Q)}, \\
\left\|b_{x t}\right\|_{L^{2}(Q)} & \leq C\left(\left\|\tilde{y}_{x}\right\|_{L^{\infty}(Q)} \cdot\left\|\tilde{y}_{t}\right\|_{L^{2}(Q)}+\left\|\tilde{y}_{x t}\right\|_{L^{2}(Q)}\right) \\
& \leq C\left\|\tilde{y}_{x}\right\|_{L^{\infty}(Q)}\left(1+\left\|\tilde{y}_{x t}\right\|_{L^{2}(Q)}\right) .
\end{aligned}
$$

We also recall that $f=m u$.

Taking into account all these facts, we obtain

$$
\begin{aligned}
\left\|y_{x}\right\|_{L^{\infty}(Q)}^{2}+\left\|y_{x t}\right\|_{L^{2}(Q)}^{2} \leq & C\left(1+\left\|\tilde{y}_{x}\right\|_{L^{\infty}(Q)}^{2}\left(1+\left\|\tilde{y}_{x t}\right\|_{L^{2}(Q)}^{2}\right)\right) \\
& \cdot\left(\int_{I}\left(b y_{x}\right)_{x}^{2}(0)+y_{x}^{2}(0) d x+\int_{Q} u_{t}^{2}+u^{2}\right), \\
\left\|\sqrt{t} y_{t}\right\|_{L^{\infty}(Q)}^{2} \leq & C\left(1+\left\|\sqrt{t} \tilde{y}_{t}\right\|_{L^{\infty}(Q)}^{2}\right)\left(1+\left\|\tilde{y}_{x}\right\|_{L^{\infty}(Q)}^{2}\left(1+\left\|\tilde{y}_{x t}\right\|_{L^{2}(Q)}^{2}\right)\right) \\
& \cdot\left(\int_{I}\left(b y_{x}\right)_{x}^{2}(0)+y_{x}^{2}(0) d x+\int_{Q} u_{t}^{2}+u^{2}\right),
\end{aligned}
$$

for all $\tilde{y}$ with $\left\|\sqrt{t} \tilde{y}_{t}\right\|_{L^{\infty}(Q)} \leq C_{2}=(1 / \mu) \max \left(1 / 2 C_{0}, 1 / 2 C_{1}\right)$, where $C_{2}$ is a constant which does not depend on $\tilde{y}, u$, or $y$.

Further combining these results, we arrive at

$$
\begin{aligned}
& \left\|y_{x}\right\|_{L^{\infty}(Q)}^{2}+\left\|\sqrt{t} y_{t}\right\|_{L^{\infty}(Q)}^{2}+\left\|y_{x t}\right\|_{L^{2}(Q)}^{2} \\
& \leq C\left(1+\left\|\sqrt{t} \tilde{y}_{t}\right\|_{L^{\infty}(Q)}^{2}\right) \\
& \quad \cdot\left(1+\left\|\tilde{y}_{x}\right\|_{L^{\infty}(Q)}^{4}+\left\|\tilde{y}_{x t}\right\|_{L^{2}(Q)}^{4}\right)\left(\int_{I}\left(b y_{x}\right)_{x}^{2}(0)+y_{x}^{2}(0) d x+\int_{Q} u_{t}^{2}+u^{2}\right) .
\end{aligned}
$$

\section{Optimization and the main result}

Now, we are ready to establish the main theorem, first for $y_{0}$ in a narrower class of functions, then for $y_{0} \in H^{1}(I)$.

Theorem 4.1. For any $\delta>0$, there exists $\eta>0$ such that, for every $y_{0} \in L^{2}(I)$ with $\left\|\left(a\left(y_{0}\right)\right)_{x x}\right\|_{L^{2}(I)}+\left\|\left(y_{0}\right)_{x}\right\|_{L^{2}(I)}+\left\|y_{0}\right\|_{L^{2}(I)} \leq \eta$, (1.1) is exactly null controllable, with a controller $u$ satisfying

$$
\int_{Q} e^{2 s(\delta-1) \alpha}\left(u^{2}+u_{t}^{2}\right) \leq C_{3}(\delta)\left\|y_{0}\right\|_{L^{2}}^{2}
$$


804 Local exact controllability of the diffusion equation in 1D

Proof. We define

$$
K=\left\{y \mid\left\|y_{x}\right\|_{L^{\infty}(Q)} \leq \rho,\left\|\sqrt{t} y_{t}\right\|_{L^{\infty}(Q)} \leq \rho,\left\|y_{x t}\right\|_{L^{2}(Q)} \leq \rho, y(0)=y_{0}\right\} .
$$

Note that this set is compact in the $L^{2}(Q)$ topology. Indeed, it can be easily seen that the set is closed, and the fact that $\|y\|_{H^{1}(Q)}$ is bounded insures its precompactness.

Consider the linearized equation (1.2) for $\tilde{y} \in K$. By the definition of $K$, for all $\tilde{y} \in K$, we have a fixed $\rho$ (which does not depend on $\tilde{y}$ ) in the Carleman inequality (Theorem 2.2). Setting a sufficiently small $\rho\left(\rho \leq C_{4}\right)$, we also find that we can apply the main result of Section 3, (3.24), for all the elements of $K$.

For brevity, in the following we will denote any constant which does not depend on $p, y, \tilde{y}, g$, and $u$ by $C$ (such a constant may, however, depend on $s, \lambda, \rho$, or $\delta)$.

Consider the optimal control problem: minimize

$$
\int_{Q} e^{-2 s \alpha} \phi^{-3} u^{2}+\epsilon^{-1} \int_{I} y^{2}(T) d x
$$

subject to (1.2). By the Pontryagin principle, this problem has a unique solution $\left(u_{\epsilon}, y_{\epsilon}\right)$. We have $u_{\epsilon}=m p_{\epsilon} e^{2 s \alpha} \phi^{3}$, where $p_{\epsilon}$ is a solution of the dual system

$$
\begin{gathered}
\left(p_{\epsilon}\right)_{t}+\left(b \cdot\left(p_{\epsilon}\right)_{x}\right)_{x}=0, \quad \forall(x, t) \in Q, \\
p_{\epsilon}(x, t)=0, \quad \forall(x, t) \in \Sigma, \\
p_{\epsilon}(x, T)=-\frac{1}{\epsilon} y_{\epsilon}(x, T), \quad \forall x \in I .
\end{gathered}
$$

Multiplying (4.4) by $y_{\epsilon}$ and (1.2) by $p_{\epsilon}$, adding the two equations together, and integrating on $Q$, we get

$$
\begin{aligned}
\int_{Q_{\omega}} e^{2 s \alpha} \phi^{3} p_{\epsilon}^{2}+\epsilon^{-1} \int_{I} y_{\epsilon}^{2}(T) d x & =-\int_{I} y_{0} p(0) d x \\
& \leq \frac{1}{2 k} \int_{I} y_{0}^{2} d x+\frac{k}{2} \int_{I} p(0)^{2} d x
\end{aligned}
$$

Applying Corollary 2.3 and choosing a small enough $k$ in (4.5), we further obtain

$$
\int_{Q_{\omega}} e^{2 s \alpha} \phi^{3} p_{\epsilon}^{2}+\epsilon^{-1} \int_{I} y_{\epsilon}^{2}(T) d x \leq C \int_{I} y_{0}^{2} d x
$$


Clearly, considering the Carleman inequality (2.2),

$$
\begin{aligned}
\int_{Q} e^{2 s(\delta-1) \alpha} u_{\epsilon}^{2} & =\int_{Q_{\omega}} e^{2 s \alpha} \phi^{3} p_{\epsilon}^{2} \cdot e^{2 s \delta \alpha} \phi^{3} \leq C \int_{Q_{\omega}} e^{2 s \alpha} \phi^{3} p_{\epsilon}^{2} \\
\int_{Q} e^{2 s(\delta-1) \alpha}\left(u_{\epsilon}\right)_{t}^{2} & =\int_{Q_{\omega}} e^{2 s(\delta-1) \alpha}\left(e^{2 s \alpha} \phi^{3}\left(p_{\epsilon}\right)_{t}+2 s e^{2 s \alpha} \alpha_{t} \phi^{3} p_{\epsilon}+3 e^{2 s \alpha} \phi^{2} \phi_{t} p_{\epsilon}\right)^{2} \\
& \leq C\left(\int_{Q} e^{2 s \alpha} \phi^{-1}\left(p_{\epsilon}\right)_{t}^{2} \cdot e^{2 s \delta \alpha} \phi^{7}+\int_{Q_{\omega}} e^{2 s \alpha} \phi^{3} p_{\epsilon}^{2} \cdot e^{2 s \delta \alpha} \phi^{7}\right) \\
& \leq C \int_{Q_{\omega}} e^{2 s \alpha} \phi^{3} p_{\epsilon}^{2} .
\end{aligned}
$$

Combining (4.7) and (4.6) gives

$$
\int_{Q} e^{2 s(\delta-1) \alpha}\left(u_{\epsilon}^{2}+\left(u_{\epsilon}\right)_{t}^{2}\right)+\epsilon^{-1} \int_{I} y_{\epsilon}^{2}(T) d x \leq C_{3} \int_{I} y_{0}^{2} d x
$$

Taking $\delta=1$ and applying (3.24) (and also considering that $\left\|\tilde{y}_{x}\right\|_{L^{\infty}(Q)} \leq \rho$, $\left.\left\|\sqrt{t} \tilde{y}_{t}\right\|_{L^{\infty}(Q)} \leq \rho,\left\|\tilde{y}_{x t}\right\|_{L^{2}(Q)} \leq \rho\right)$ give

$$
\begin{aligned}
& \left\|\left(y_{\epsilon}\right)_{x}\right\|_{L^{\infty}(Q)}^{2}+\left\|\sqrt{t}\left(y_{\epsilon}\right)_{t}\right\|_{L^{\infty}(Q)}^{2}+\left\|\left(y_{\epsilon}\right)_{x t}\right\|_{L^{2}(Q)}^{2} \\
& \leq C\left(1+\rho^{2}\right)\left(1+2 \rho^{4}\right)\left(\int_{I}\left(b \cdot\left(y_{0}\right)_{x}\right)_{x}^{2}+\left(y_{0}\right)_{x}^{2} d x+\int_{Q}\left(u_{\epsilon}\right)_{t}^{2}+u_{\epsilon}^{2}\right) \\
& \leq C_{4}\left(1+\rho^{6}\right) \int_{I}\left(b \cdot\left(y_{0}\right)_{x}\right)_{x}^{2}+\left(y_{0}\right)_{x}^{2}+y_{0}^{2} d x
\end{aligned}
$$

Furthermore, since $b(0)=a^{\prime}(\tilde{y}(0))=a^{\prime}\left(y_{0}\right)$, we obtain

$$
\int_{I}\left(b \cdot\left(y_{0}\right)_{x}\right)_{x}^{2}+\left(y_{0}\right)_{x}^{2}+y_{0}^{2} d x=\int_{I}\left(a\left(y_{0}\right)\right)_{x x}^{2}+\left(y_{0}\right)_{x}^{2}+y_{0}^{2} d x
$$

hence the condition imposed on $y_{0}$ in the statement of the theorem. Thus we get

$$
\begin{aligned}
\left\|\left(y_{\epsilon}\right)_{x}\right\|_{L^{\infty}(Q)}^{2} & +\left\|\sqrt{t}\left(y_{\epsilon}\right)_{t}\right\|_{L^{\infty}(Q)}^{2}+\left\|\left(y_{\epsilon}\right)_{x t}\right\|_{L^{2}(Q)}^{2} \\
\leq & C_{4}\left(1+\rho^{6}\right) \int_{I}\left(a\left(y_{0}\right)\right)_{x x}^{2}(0)+\left(y_{0}\right)_{x}^{2}+y_{0}^{2} d x
\end{aligned}
$$

By making

$$
\int_{I}\left(a\left(y_{0}\right)\right)_{x x}^{2}+\left(y_{0}\right)_{x}^{2}+y_{0}^{2} d x<\eta
$$

for a sufficiently small $\eta$ (such as $\left.\eta=\rho / C_{4}\left(1+\rho^{6}\right)\right)$, we obtain that $y_{\epsilon} \in K$. 
In conclusion, by (4.8) with $\delta=1, u_{\epsilon}$ and $\left(u_{\epsilon}\right)_{t}$ belong to a bounded set in $L^{2}(Q)$, the same for every $\epsilon$, and we have also proved that $y_{\epsilon}$ belongs to $K$, a set that is compact in $L^{2}(Q)$.

Choose a sequence of $\left(u_{\epsilon}, y_{\epsilon}\right), \epsilon \rightarrow 0$, that achieves the optimum in expression (4.3). From the above, it follows that (on a subsequence)

$$
\begin{aligned}
u_{\epsilon} \longrightarrow u & \text { weakly in } L^{2}(Q), \\
\left(u_{\epsilon}\right)_{t} \longrightarrow v & \text { weakly in } L^{2}(Q), \\
y_{\epsilon} \longrightarrow y & \text { strongly in } L^{2}(Q) .
\end{aligned}
$$

Obviously, $v=u_{t}$. Other immediate consequences are that $\left(y_{\epsilon}\right)_{t}$ tends to $y_{t}$ in $H^{-1}(Q)$ and that $\left(b\left(y_{\epsilon}\right)_{x}\right)_{x}$ tends to $\left(b y_{x}\right)_{x}$ in $L^{2}\left((0, T) ; H^{-2}(I)\right)$; then $(u, y)$ satisfies the linearized equation (1.2). Furthermore, the strong convergence of $y_{\epsilon}$ implies that $y \in K$. Last but not least, by making $\epsilon \rightarrow 0$ in (4.6), we see that $y(T) \equiv 0$.

As for $\left\|e^{s(1-\delta) \alpha} u\right\|_{L^{2}(Q)}$ and $\left\|e^{s(1-\delta) \alpha} u_{t}\right\|_{L^{2}(Q)}$, we recall that, for any sequence $\left(f_{n}\right)_{n \in \mathbb{N}}$ that converges weakly to $f$ in $L^{2}$, we have $\|f\|_{L^{2}(Q)} \leq \liminf \left\|f_{n}\right\|_{L^{2}(Q)}$.

Thus, for any auxiliary function $\tilde{y} \in K$, we have obtained a pair $(y, u)$ that satisfies system (1.2), with $y \in K, y(T) \equiv 0$, and

$$
\int_{Q} e^{2 s(\delta-1) \alpha}\left(u^{2}+u_{t}^{2}\right) \leq C_{3}\left\|y_{0}\right\|_{L^{2}}^{2}
$$

We are now ready to apply Kakutani's theorem. Consider $\Phi: K \rightarrow 2^{K}$,

$$
\begin{aligned}
\Phi(\tilde{y})= & \left\{y \mid y \in K, y(T) \equiv 0 \text {, and } \exists u \in H^{1}\left([0, T] ; L^{2}(I)\right),\right. \\
& \text { with } \left.\int_{Q} e^{2 s(\delta-1) \alpha}\left(u^{2}+u_{t}^{2}\right) \leq C_{3}\left\|y_{0}\right\|_{L^{2}} \text {, such that }(u, y) \text { satisfies }(1.2)\right\} .
\end{aligned}
$$

Clearly $\Phi$ is well defined, takes nonempty values for every $\tilde{y} \in K$, and has convex values.

In order to prove that $\Phi$ has closed values, first we fix a $\tilde{y}$, consider a convergent sequence

$$
y_{n} \longrightarrow y \quad \text { in the } L^{2}(Q) \text { norm, }
$$

with $y_{n} \in \Phi(\tilde{y})$, and choose the corresponding $u_{n}$ as in the definition of $\Phi$ (4.15). Since $y_{n} \in K$, for all $n$, and $K$ is compact, it follows that their limit $y$ 
is also in $K$. Furthermore,

$$
\begin{aligned}
\left\|y_{n}(T)-y(T)\right\|_{L^{2}(I)} & \leq\left\|y_{n}-y\right\|_{C\left([0, T] ; L^{2}(I)\right)} \\
& \leq\left\|y_{n}-y\right\|_{H^{1}\left([0, T] ; L^{2}(I)\right)}^{1 / 2}\left\|y_{n}-y\right\|_{L^{2}(Q)}^{1 / 2} \\
& \leq C \rho^{1 / 2} \cdot\left\|y_{n}-y\right\|_{L^{2}(Q)}^{1 / 2},
\end{aligned}
$$

so $y(T) \equiv 0$.

Since $\int_{Q} e^{2 s(\delta-1) \alpha}\left(u_{n}^{2}+\left(u_{n}\right)_{t}^{2}\right) \leq C_{3}\left\|y_{0}\right\|_{L^{2}(I)}^{2}$, we may select a weakly convergent subsequence which tends to a limit $u$ with $\int_{Q} e^{2 s(\delta-1) \alpha}\left(u^{2}+u_{t}^{2}\right) \leq C_{3}\left\|y_{0}\right\|_{L^{2}(I)}^{2}$ (the proof is as above). It can be seen that $\left(y_{n}\right)_{t} \rightarrow y_{t}$ strongly in the $H^{-1}(Q)$ norm and $\left(b \cdot\left(y_{n}\right)_{x}\right)_{x} \rightarrow\left(b y_{x}\right)_{x}$ strongly in the $L^{2}\left((0, T) ; H^{-2}(I)\right)$ norm. By passing to the weak limit in (1.2), satisfied by $\left(u_{n}, y_{n}\right)$, we obtain that $(u, y)$ also satisfies the equation for the same $\tilde{y}$. Therefore, $y \in \Phi(\tilde{y})$, and thus $\Phi(\tilde{y})$ is closed; in fact, since $\Phi(\tilde{y}) \subset K$, it follows that $\Phi(\tilde{y})$ is compact for every $\tilde{y} \in K$.

In such a case, the lower semicontinuity of $\Phi$ can be obtained from the fact that it has a closed graph. Indeed, if $\tilde{y}_{n} \in K, \tilde{y}_{n} \rightarrow \tilde{y}$, and $y_{n} \in \Phi\left(\tilde{y}_{n}\right) \rightarrow y$ in the $L^{2}(Q)$ norm, consider the corresponding $u_{n}$, as above, and we obtain (on a subsequence) that

$$
\begin{aligned}
& u_{n} \longrightarrow u \quad \text { weakly in } L^{2}(Q) \text {, } \\
& \left(u_{n}\right)_{t} \longrightarrow u_{t} \quad \text { weakly in } L^{2}(Q) \text {, with } \int_{Q} e^{2 s(\delta-1) \alpha}\left(u^{2}+u_{t}^{2}\right) \leq C_{3}\left\|y_{0}\right\|_{L^{2}} ; \\
& \left(y_{n}\right)_{t} \longrightarrow y_{t} \quad \text { strongly in } H^{-1}(Q) ; \\
& \left(y_{n}\right)_{x} \longrightarrow y_{x} \quad \text { strongly in } L^{2}\left((0, T) ; H^{-1}(I)\right) \text {; } \\
& \tilde{y}_{n} \longrightarrow \tilde{y} \quad \text { strongly in } L^{2}(Q) .
\end{aligned}
$$

Since $\left|a^{\prime}\left(\tilde{y}_{n}\right)-a^{\prime}(\tilde{y})\right| \leq C\left|y_{n}-y\right|$, it also follows that $a^{\prime}\left(\tilde{y}_{n}\right) \rightarrow a^{\prime}(\tilde{y})$ strongly in $L^{2}(Q)$ (and weakly-star in $L^{\infty}(Q)$ ). Then $a^{\prime}\left(\tilde{y}_{n}\right)\left(y_{n}\right)_{x} \rightarrow a^{\prime}(\tilde{y}) y_{x}$ weakly in $L^{2}\left((0, T) ; H^{-1}(I)\right)$, or, equivalently, $\left(a^{\prime}\left(\tilde{y}_{n}\right)\left(y_{n}\right)_{x}\right)_{x} \rightarrow\left(a^{\prime}(\tilde{y}) y_{x}\right)_{x}$ weakly in $L^{2}\left((0, T) ; H^{-2}(I)\right)$. By going to the weak limit in (1.2), we obtain that the pair $(u, y)$ also satisfies the linearized system, with $b=a^{\prime}(\tilde{y})$. The other conditions $(\tilde{y}, y \in K, y(T) \equiv 0)$ are obviously satisfied (see above the details of the proof), so $(\tilde{y}, y)$ belongs to the graph of $\Phi$.

Thus we can apply Kakutani's theorem and obtain that there is $y \in K$ such that $y \in \Phi(y)$. Such a $y$ is a solution of the diffusion equation (1.1) with $y(0)=y_{0}$ and $y(T) \equiv 0$. In addition, its controller $u$ satisfies the required estimate.

Now, we come back to the more general case, $y_{0} \in H^{1}(I)$. 
Theorem 4.2. For any $\delta>0$, there exists $\eta>0$ such that, for every $y_{0} \in H^{1}(I)$ with $\left\|y_{0}\right\|_{H^{1}(I)} \leq \eta$, (1.1) is exactly null controllable, with a controller $u$ satisfying

$$
\int_{Q} e^{2 s(\delta-1) \alpha}\left(u^{2}+u_{t}^{2}\right) \leq C_{3}\left\|y_{0}\right\|_{L^{2}(I)}^{2}
$$

Proof. We are going to divide the interval $[0, T]$ into two parts by choosing $0<T_{0}<T$. On the first part of the interval, we will make $u \equiv 0$; the equation becomes

$$
\begin{aligned}
y_{t}-\left(b y_{x}\right)_{x} & =0, \quad \forall(x, t) \in Q, \\
y(x, t) & =0, \quad \forall(x, t) \in \Sigma, \\
y(x, 0) & =y_{0}, \quad x \in I,
\end{aligned}
$$

where we have renamed $I \times\left[0, T_{0}\right] \equiv Q_{T_{0}} \equiv Q$ and $\partial I \times\left[0, T_{0}\right] \equiv \Sigma$ for greater convenience. We will use the regularizing properties of (4.20), eventually obtaining that

$$
\int_{I}(a(y))_{x x}^{2}\left(T_{0}\right)+y_{x}^{2}\left(T_{0}\right)+y^{2}\left(T_{0}\right) d x \leq C \int_{I} y_{0}^{2}+y_{x}^{2}(0) d x .
$$

Then, applying Theorem 4.1, we will be able to establish the null controllability of $y$ on the interval $\left[T_{0}, T\right]$. Finally, we note that the function $u$ defined by

$$
u(x, t)= \begin{cases}0, & \text { for } t \in\left[0, T_{0}\right] \\ u^{*}(x, t), & \text { for } t \in\left[T_{0}, T\right]\end{cases}
$$

where $u^{*}$ is a controller for $y$ on $\left[T_{0}, T\right]$, still has all the desired properties (it belongs to $H^{1}\left([0, T] ; L^{2}(I)\right)$ and it satisfies (4.19)). Then the proof is complete.

Now, we establish estimate (4.21). For the beginning, we will only assume in (4.20) that $b_{t} \in L^{2}(Q)$ and $b_{x} \in L^{\infty}\left(\left[0, T_{0}\right] ; L^{2}(I)\right)$.

Multiplying (4.20) by $y$ and integrating on $Q_{t}$, we get

$$
\int_{I} y^{2}(t) d x+2 \int_{Q_{t}} b y_{x}^{2}=\int_{I} y^{2}(0) d x
$$

Multiplying the same equation by $y_{t}$ and integrating by parts for $t$, we get

$$
\int_{Q_{t}} y_{t}^{2}+\frac{1}{2} \int_{I} b y_{x}^{2}(t)=\frac{1}{2} \int_{I} b y_{x}^{2}(0)+\frac{1}{2} \int_{Q_{t}} b_{t} y_{x}^{2} .
$$

Then, it is true that

$$
\int_{Q_{t}} y_{t}^{2}+\int_{I} y_{x}^{2}(t) d x \leq C\left(\int_{I} y_{x}^{2}(0) d x+\left(\int_{Q_{t}} b_{t}^{2}\right)^{1 / 2} \cdot\left(\int_{Q_{t}} y_{x}^{4}\right)^{1 / 2}\right),
$$


and, consequently, ( since $0 \leq t \leq T_{0}$ )

$$
\begin{aligned}
& \int_{Q} y_{t}^{2}+\sup _{t \in\left[0, T_{0}\right]} \int_{I} y_{x}^{2}(t) d x \\
& \quad \leq C\left(\int_{I} y_{x}^{2}(0) d x+\left\|b_{t}\right\|_{L^{2}(Q)} \cdot\left\|y_{x}\right\|_{L^{2}\left(\left(0, T_{0}\right) ; L^{\infty}(I)\right)} \cdot \sup _{t \in\left[0, T_{0}\right]}\left\|y_{x}(t)\right\|_{L^{2}(I)}\right) .
\end{aligned}
$$

We note that $\left\|y_{x}\right\|_{L^{2}\left(\left(0, T_{0}\right) ; L^{\infty}(I)\right)} \leq C\left\|\left(b y_{x}\right)_{x}\right\|_{L^{2}(Q)}=C\left\|y_{t}\right\|_{L^{2}(Q)}$, so

$$
\begin{aligned}
& \int_{Q} y_{t}^{2}+\sup _{t \in\left[0, T_{0}\right]} \int_{I} y_{x}^{2}(t) d x \\
& \quad \leq C_{5}\left(\int_{I} y_{x}^{2}(0) d x+\left\|b_{t}\right\|_{L^{2}(Q)}\left(\int_{Q} y_{t}^{2}+\sup _{t \in\left[0, T_{0}\right]} \int_{I} y_{x}^{2}(t) d x\right)\right) .
\end{aligned}
$$

Making $\left\|b_{t}\right\|_{L^{2}(Q)}$ sufficiently small (e.g., $\left\|b_{t}\right\|_{L^{2}(Q)} \leq 1 / 2 C_{5}$ ), we obtain

$$
\int_{Q} y_{t}^{2}+\sup _{t \in\left[0, T_{0}\right]} \int_{I} y_{x}^{2}(t) d x \leq C \int_{I} y_{x}^{2}(0) d x
$$

As an immediate consequence, we also get $\int_{Q}\left(b y_{x}\right)_{x}^{2} \leq C \int_{I} y_{x}^{2}(0)$ (this will be useful later on).

Rewriting (4.28) and recalling that, by definition, $b=a^{\prime}(\tilde{y})$, we obtain

$$
\left\|y_{t}\right\|_{L^{2}(Q)}+\sup _{t \in\left[0, T_{0}\right]}\left\|y_{x}(t)\right\|_{L^{2}(I)} \leq C_{6}\left\|y_{x}(0)\right\|_{L^{2}(I)}
$$

if $\left\|\tilde{y}_{t}\right\|_{L^{2}(Q)} \leq 1 / 2 M C_{5}$.

Consider the set

$$
K=\left\{y \mid\left\|y_{t}\right\|_{L^{2}(Q)}+\sup _{t \in\left[0, T_{0}\right]}\left\|y_{x}(t)\right\|_{L^{2}(I)} \leq \frac{1}{2 M C_{5}}, y(0)=y_{0}\right\},
$$

which is clearly compact in $L^{2}(Q)$ (being closed in $L^{2}(Q)$ and bounded in $H^{1}(Q)$ ). Then, define $\Phi: K \rightarrow 2^{K}$ by

$$
\Phi(\tilde{y})=\{y \mid y \in K, y \text { is a solution of }(4.20)\}
$$

For $\left\|y_{x}(0)\right\|_{L^{2}(I)} \leq 1 /\left(C_{6} \cdot 2 M C_{5}\right)$, the multifunction $\Phi$ has nonempty values everywhere. It can be easily checked that it also has convex values. 
Consider a sequence of solutions $y_{n} \in \Phi(\tilde{y})$ which converges in $L^{2}(Q)$ to a limit $y$. Then, $\left(y_{n}\right)_{t} \rightarrow y_{t}$ in $H^{-1}(Q)$ and $\left(b \cdot\left(y_{n}\right)_{x}\right)_{x} \rightarrow\left(b y_{x}\right)_{x}$ in $L^{2}\left(\left(0, T_{0}\right)\right.$; $\left.H^{-2}(I)\right)$. By going to the limit in (4.20) for $y_{n}$, we obtain that $y_{t}-\left(b y_{x}\right)_{x}=0$ in the weak sense (in $H^{-2}(Q)$ ), and therefore $y \in \Phi(\tilde{y})$. Thus we have proved that $\Phi$ has closed values (compact, as a matter of fact).

All that is left is to show that $\Phi$ has a closed graph (and thus is upper semicontinuous). Indeed, if we take two sequences $y_{n}$ and $\tilde{y}_{n}, y_{n} \in \Phi\left(\tilde{y}_{n}\right)$, converging to $y$ and to $\tilde{y}$, respectively, we successively obtain, in the same manner as in the proof of Theorem 4.1, that

$$
\begin{array}{ll}
\left(y_{n}\right)_{t} \longrightarrow y_{t} & \text { in } H^{-1}(Q), \\
\left(y_{n}\right)_{x} \longrightarrow y_{x} & \text { in } L^{2}\left(\left(0, T_{0}\right) ; H^{-1}(I)\right), \\
\tilde{y}_{n} \longrightarrow \tilde{y} & \text { in } L^{2}(Q), \\
a^{\prime}\left(\tilde{y}_{n}\right) \longrightarrow a^{\prime}(\tilde{y}) & \text { weakly-star in } L^{\infty}(Q), \\
a^{\prime}\left(\tilde{y}_{n}\right) \cdot\left(y_{n}\right)_{x} \longrightarrow a^{\prime}(\tilde{y}) y_{x} & \text { in } L^{2}\left(\left(0, T_{0}\right) ; H^{-1}(I)\right), \\
\left(a^{\prime}\left(\tilde{y}_{n}\right) \cdot\left(y_{n}\right)_{x}\right)_{x} \longrightarrow\left(a^{\prime}(\tilde{y}) y_{x}\right)_{x} & \text { in } L^{2}\left(\left(0, T_{0}\right) ; H^{-2}(I)\right) .
\end{array}
$$

By going to the limit in (4.20), satisfied by all the functions $y_{n}$ with $b=a^{\prime}\left(\tilde{y}_{n}\right)$, we obtain that $y \in \Phi(\tilde{y})$, so the graph is closed.

Now, all the conditions needed to apply Kakutani's theorem are fulfilled and we obtain that $\Phi$ has a fixed point $y \in \Phi(y)$, and therefore the diffusion equation

$$
\begin{gathered}
y_{t}-(a(y))_{x x}=0, \quad \forall(x, t) \in Q=I \times\left(0, T_{0}\right), \\
y(x, t)=0, \quad \forall(x, t) \in \Sigma=\partial I \times\left(0, T_{0}\right), \\
y(x, 0)=y_{0}, \quad x \in I,
\end{gathered}
$$

has a solution $y$ with

$$
\left\|y_{t}\right\|_{L^{2}(Q)}+\sup _{t \in\left[0, T_{0}\right]}\left\|y_{x}(t)\right\|_{L^{2}(I)} \leq \underbrace{\frac{1}{2 M C_{5}}}_{C}\left\|y_{x}(0)\right\|_{L^{2}(I)},
$$

for $\left\|y_{x}(0)\right\|_{L^{2}(I)} \leq 1 /\left(C_{6} \cdot 2 M C_{5}\right)$.

However, the solution of (4.33) has further regularity properties, of which we are going to employ only one. For convenience, we will keep the notation $b=a^{\prime}(\tilde{y}) \equiv a^{\prime}(y)$. By multiplying (3.1) by $\left(b y_{x}\right)_{x t}$, we get

$$
\int_{Q_{t}}\left(b y_{x}\right)_{x}\left(b y_{x}\right)_{x t}+\int_{Q_{t}} b y_{x t}^{2}+\int_{Q_{t}} y_{x t} b_{t} y_{x}=0
$$

hence

$$
\frac{1}{2} \int_{I} t\left(b y_{x}\right)_{x}^{2}(t) d x+\int_{Q_{t}} t b y_{x t}^{2}+\int_{Q_{t}} t y_{x t} b_{t} y_{x}=\frac{1}{2} \int_{Q_{t}}\left(b y_{x}\right)_{x}^{2} .
$$


In this equation, we have

$$
\left|\int_{Q_{t}} t y_{x t} b_{t} y_{x}\right| \leq \frac{\mu}{2} \int_{Q_{t}} t y_{x t}^{2}+\frac{1}{2 \mu} \int_{Q_{t}} t b_{t}^{2} y_{x}^{2}
$$

where we may take $\mu$ to be the same constant as in the beginning of the paper $\left(\mu \leq a^{\prime}(x)\right.$, for all $x \in \mathbb{R}$, so that $\left.\mu \leq a^{\prime}(y)=b\right)$. Then,

$$
\int_{Q_{t}} t b_{t}^{2} y_{x}^{2} \leq \int_{Q} b_{t}^{2} \cdot\left\|t b y_{x}^{2}\right\|_{L^{\infty}(Q)} \leq C \int_{Q} y_{t}^{2} \cdot \sup _{t \in\left[0, T_{0}\right]} \int_{I} t\left(b y_{x}\right)_{x}^{2}(t) d x .
$$

Consequently, we obtain (by using evaluation (4.34) for $\left.\left\|y_{t}\right\|_{L^{2}(Q)}\right)$ that

$$
\begin{aligned}
& \sup _{t \in\left[0, T_{0}\right]} \int_{I} t\left(b y_{x}\right)_{x}^{2}(t) d x+\int_{Q} t y_{x t}^{2} \\
& \quad \leq C_{7}\left(1+\sup _{t \in\left[0, T_{0}\right]} \int_{I} t\left(b y_{x}\right)_{x}^{2}(t) d x\right) \int_{I} y_{x}^{2}(0) d x, \quad \forall t \in\left[0, T_{0}\right] .
\end{aligned}
$$

Again, for sufficiently small $\left\|y_{x}(0)\right\|_{L^{2}(I)}$ (such as $\left.\left\|y_{x}(0)\right\|_{L^{2}(I)}^{2} \leq 1 / 2 C_{7}\right)$, we have proved that

$$
\sup _{t \in\left[0, T_{0}\right]} \int_{I} t\left(b y_{x}\right)_{x}^{2}(t) d x+\int_{Q} t y_{x t}^{2} \leq C \int_{I} y_{x}^{2}(0) d x
$$

By choosing $t=T_{0}$ in (4.23), (4.34), and (4.40), we obtain

$$
\int_{I}(a(y))_{x x}^{2}\left(T_{0}\right)+y_{x}^{2}\left(T_{0}\right)+y^{2}\left(T_{0}\right) d x \leq C\left(\int_{I} y_{0}^{2} d x+\int_{I} y_{x}^{2}(0) d x\right),
$$

for any $y_{0}$ with $\left\|\left(y_{0}\right)_{x}\right\|_{L^{2}(I)}$ sufficiently small. The proof of Theorem 4.2 is thus concluded.

\section{References}

[1] V. Barbu, Controllability of parabolic and Navier-Stokes equations, Sci. Math. Jpn. 56 (2002), no. 1, 143-211.

[2] A. V. Fursikov and O. Yu. Imanuvilov, Controllability of Evolution Equations, Lecture Notes Series, vol. 34, Seoul National University, Research Institute of Mathematics, Global Analysis Research Center, Seoul, 1996.

Marius Beceanu: Department of Mathematics, Princeton University, Princeton, NJ 0544, USA

E-mail address: mbeceanu@princeton.edu 


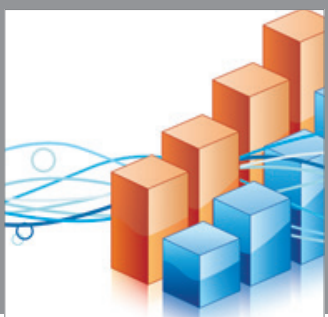

Advances in

Operations Research

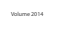

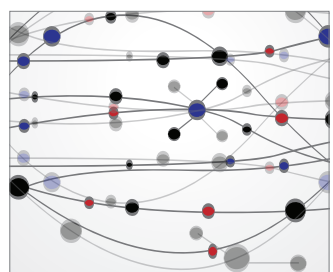

\section{The Scientific} World Journal
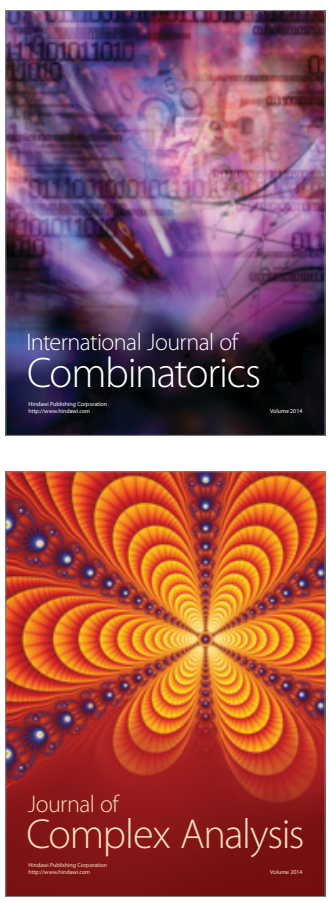

International Journal of

Mathematics and

Mathematical

Sciences
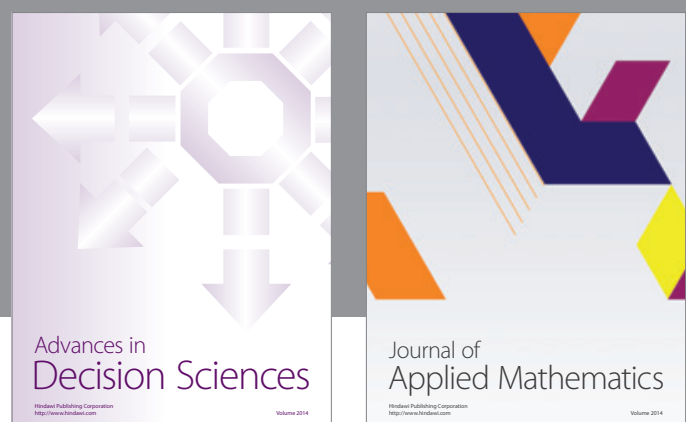

Journal of

Applied Mathematics
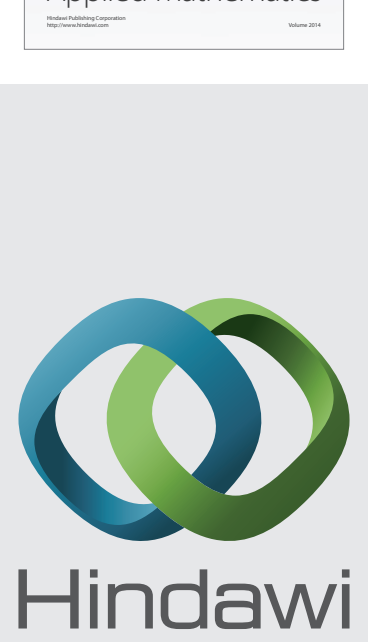

Submit your manuscripts at http://www.hindawi.com
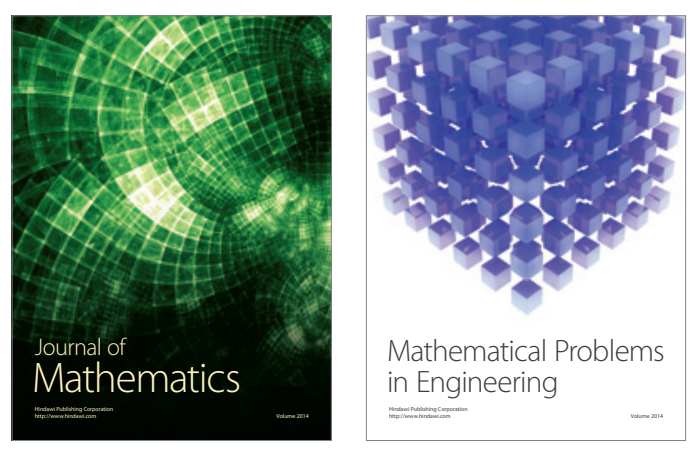

Mathematical Problems in Engineering
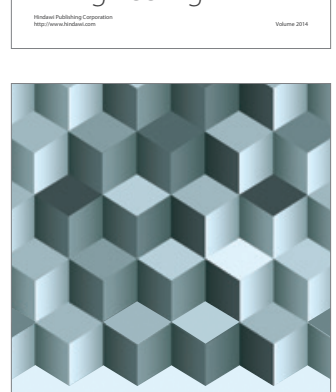

Journal of

Function Spaces
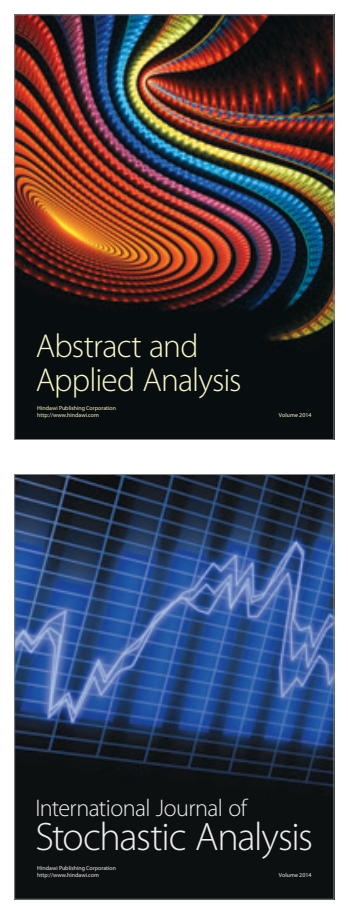

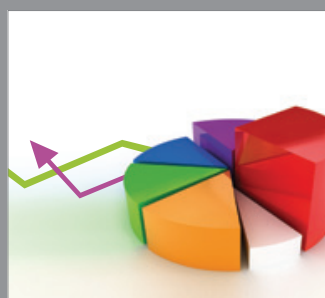

ournal of

Probability and Statistics

Promensencen
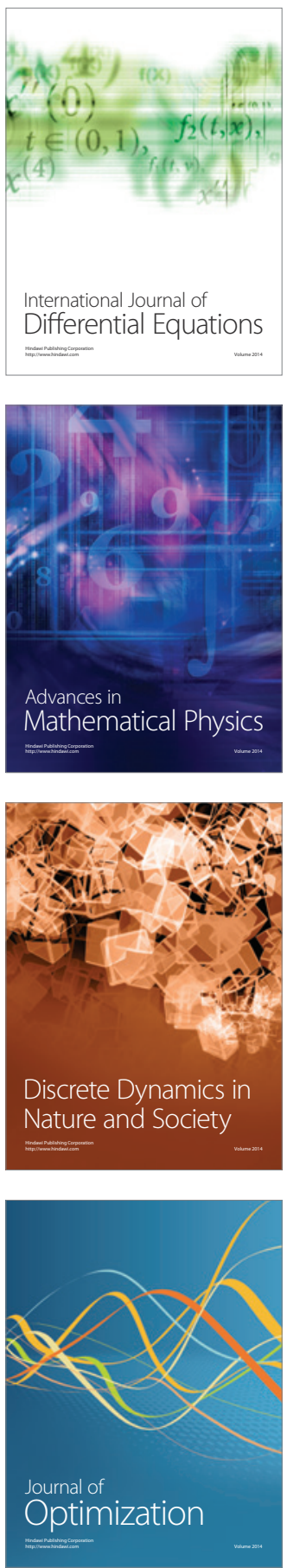